\title{
Essais
}

Revue interdisciplinaire d'Humanités

\section{Prêter attention aux mondes}

Vers une écologie décentrée, plurielle et interprétative

Fabien Colombo, Nestor Engone Elloué et Bertrand Guest

\section{OpenEdition}

Journals

Édition électronique

URL : http://journals.openedition.org/essais/435

DOI : 10.4000/essais.435

ISSN : 2276-0970

Éditeur

École doctorale Montaigne Humanités

\section{Édition imprimée}

Date de publication : 1 janvier 2018

Pagination : 7-16

ISBN : 979-10-97024-03-1

ISSN : 2417-4211

\section{Référence électronique}

Fabien Colombo, Nestor Engone Elloué et Bertrand Guest, «Prêter attention aux mondes », Essais [En ligne], 13 | 2018, mis en ligne le 01 décembre 2019, consulté le 24 septembre 2020. URL : http:// journals.openedition.org/essais/435; DOI : https://doi.org/10.4000/essais.435 


\section{Prêter attention aux mondes \\ Vers une écologie décentrée, plurielle \\ et interprétative}

\section{Avant-propos}

\section{Fabien Colombo, Nestor Engone Elloué, Bertrand Guest}

\section{À Laurent Leylavergne, in memoriam.}

" $\mathrm{Si}$ un lion pouvait parler, nous ne pourrions pas le comprendre ${ }^{1}$.»

Les études de l'homme et de la nature n'ont eu de cesse de se séparer alors que les savoirs se transformaient en disciplines de plus en plus spécialisées mais lointaines les unes des autres. Née dans le contexte positiviste au sein de ces sciences qui, devenant modernes, s'étaient coupées des classiques humanités, l'écologie fut longtemps délaissée par ces dernières jusqu'à un passé récent. Elle procède de la nécessité d'étudier, à nouveau, homme et nature ensemble.

En œuvrant à concilier le souci descriptif de la nature, celui, prescriptif, des façons de vivre en bonne intelligence avec elle, ainsi que celui, esthétique, d'une forme d'écriture lisible par tous, des scientifiques comme Aldo Leopold (Almanach d'un comté des sables, 1949) et Rachel Carson (Printemps silencieux, 1962) ont pleinement participé par leurs écrits, à construire une passerelle unissant la connaissance scientifique de la nature et la pratique, linguistique et éthique, des humanités. Emprunter cette passerelle suppose de sortir de l'enfermement disciplinaire et de se saisir de l'écologie comme d'un objet d'étude, à la manière dont s'y est pris Arne Naess, l'un des premiers à avoir formulé les principes d'une philosophie de l'écologie au début des années 1970. Cette date marque d'ailleurs un tournant épistémologique qui a vu un nombre croissant de sciences humaines et sociales emprunter cette passerelle en prenant l'écologie ou l'environnement pour objet ${ }^{2}$.

1 Ludwig Wittgenstein, Philosophische Untersuchungen (Investigations philosophiques), Frankfurt/ Main, Suhrkamp, 1967, II, XI, p. 536. „Könnte ein Löwe sprechen, wir könnten ihn nicht verstehen."

2 C'est aussi la thèse défendue par Guillaume Blanc, Élise Demeulenaere et Wolf Feuerhahn 
Avec l'engouement suscité par les rencontres interétatiques qui se sont déroulées ces quarante dernières années, de la Conférence des Nations unies sur l'environnement à Stockholm en 1972, à la Conférence de Paris sur le Climat en 2015 (COP21), l'intérêt du maintien de cette passerelle entre sciences, droit et humanités se justifie par la nécessité d'encadrer la prise de décision, d'en apprécier les nombreux enjeux sociaux, moraux et esthétiques. Cela suppose de poser la question de l'écologie depuis un faisceau de disciplines réunies dans ce but, réunies fortement, jusqu’à accepter leur reconfiguration ; la poser avec recul, hors de l'urgence qui lui semble inéluctablement (et dommageablement) attachée dans les discours médiatiques.

Lécologie politique comme l'écologie scientifique reconnaissent désormais de plus en plus leur dialogue et leur recours nécessaires à l'histoire et aux sciences sociales, mais aussi à la littérature, à la philosophie, aux arts, aux mythes et aux langues. Autant de disciplines qui pourtant, lorsqu'elles sont associées à un champ où dominent les sciences dures et expérimentales, ne le sont bien souvent qu'à la marge, et rarement en tant que creuset épistémologique. Il ne va d'ailleurs plus de soi, c'est peu dire, que celles-ci se conçoivent comme " humanités ».

C'est dans un double sens que nous lisons la notion d' " humanités ». D'une part, elle peut renvoyer à l'agencement épistémologique en pleine refondation de l'étude et du renouveau traductif des langues anciennes à la Renaissance. Ce terme désigne encore l'alliance contemporaine des langues, des arts, des lettres et des sciences humaines et sociales, domaines du savoir encore trop en marge des questionnements écologiques. D'autre part, au pluriel, cette notion fait référence au genre humain et permet d'attirer l'attention sur les inégalités sociales et historiques ainsi que sur les disparités de visions du monde qui structurent cette " unité dans la diversité ${ }^{3}$ " qu'est l'humain, au même titre que la nature. Nous nous sommes placés dans le projet des Humanités environnementales, qui « au lieu d'envisager une nature physique associée à une culture humaine distincte [...], fondent leur approche sur les ontologies interconnectées, à savoir un ensemble de réseaux associant les êtres humains et non-humains ${ }^{4} »$. Il y est question de penser une humanité plurielle et une pluralité des histoires humaines pour répondre à l'illusion d'un "Homme » unique, véhiculée par la récente mise en avant de la notion controversée d'Anthropocène.

dans l'ouvrage qui vient de paraittre sous leur direction : Humanités environnementales. Enquêtes et contre-enquêtes, Paris, Publications de la Sorbonne, 2017.

3 Alexander von Humboldt, Kosmos, Entwurf einer physischen Weltbeschreibung [1834-1859], Hans Magnus Enzensberger (ed.), Frankfurt am Main, Eichborn Verlag, 2004, p. 10.

4 Portail des Humanités Environnementales : http://humanitesenvironnementales.fr/fr/les-ressources, consulté le 20 avril 2017. 
Certains comme Dipesh Chakrabarty, souhaitent rappeler que cette hypothétique nouvelle ère de l'histoire de la Terre, caractérisée par l'impact géologique des facteurs humains, est "l'histoire de la démocratisation de la consommation" ", et de ce fait, l'histoire de l'espèce humaine dans son ensemble. D'autres comme Christophe Bonneuil et Jean-Baptiste Fressoz ${ }^{6}$ préferrent au contraire défaire le récit dominant de l'Anthropocène et le remplacent par l'histoire des inégalités d'impacts. Ils soutiennent à cet effet que l'Anthropocène est un "Anglocène ${ }^{7}$ " et relèvent à cet effet l'impact et le rôle significatif qu'ont joué le Royaume-Uni et les États-Unis dans la dégradation de l'environnement mondial. Quoi qu'il en soit, le mérite épistémologique de la notion d'Anthropocène est sans doute de rendre nécessaire un nouveau matérialisme réellement transdisciplinaire, à même de réécrire, en les (re)connectant, deux histoires distinguées à tort. Sans aucun déterminisme univoque de part et d'autre, il nous rappelle en effet que l'évolution physique des roches, des sciences et des techniques ne peut plus être pensée en dehors de l'histoire des sociétés, de l'économie et de la politique, pas plus qu'en dehors de celle des idées, des formes expressives, des imaginaires, des poétiques et des cultures, des langues enfin, mémoires des paysages et des multiples rapports ayant existé entre hommes et terres. Avec ce récit d'un basculement du système Terre dans une ère nouvelle et inconnue, les sciences de l'Homme et les sciences de la Nature repensent leurs rapports autrement que par la démarcation mutuelle qui fut le pli "moderne ", sans doute en faisant droit au point de vue des « terriens ${ }^{8}$ ".

Mais de même que la notion d'humanités en ce deuxième sens de genre humain, l'écologie suppose aussi d'être déclinée au pluriel. Comme l'a montré dès 1989 Félix Guattari qui a développé dans cette pluralité même la notion d'écosophie, l'écologie se déploie toujours sur les plans trop souvent distincts de l'environnement, de la société et de la production des subjectivités humaines ${ }^{9}$. Depuis lors, la montée en puissance du " capitalisme vert " et d'un solutionnisme technique aux crises environnementales semble avoir capté l'usage public de la notion d' " écologie " à mesure qu'elle s'est institutionnalisée en une préoccupation sectorielle dépolitisée ${ }^{10}$, confiée à des experts

5 Dipesh Chakrabarty, "Quelques failles dans la pensée sur le changement climatique ", in Émilie Hache (éd.), De l'univers clos au monde infini, Bellevaux, Dehors, 2014, p. 135.

6 Cf. Christophe Bonneuil, Jean-Baptiste Fressoz. L'Évènement Anthropocène. La Terre, l'histoire et nous, Paris, Seuil, 2013.

7 Ibid., p. 134.

8 Cf. Bruno Latour, Face à Gä̈a. Huit conférences sur le nouveau régime climatique, Paris, La Découverte, 2015.

9 Félix Guattari, Les trois écologies, Paris, Galilée, 1989.

10 Cf. Catherine et Raphaël Larrère, Du bon usage de la nature. Pour une philosophie de l'environnement, chap. IV, "Protéger la nature : une tâche moderne ", Paris, Flammarion, 1997, p. 175-206. 
techniques et financiers (protection d'espèces, d'espaces et de paysages choisis, marché du carbone, ingénierie climatique...).

Cette évolution conditionne celle des mots mêmes de l'écologie, souvent détournés au prix d'un immense malentendu en arguments de vente aptes à conforter paradoxalement cette prédation que dénonce l'écologie politique. D'où la distinction faite par Arne Naess entre " écologie superficielle " et " écologie profonde ${ }^{11}$ ", ou celle proposée plus récemment par Yves Citton qui différencie " écologie gestionnaire " et " écologie radicale " : la première entend " économiser nos ressources afin de produire de façon plus soutenable les modes de vie qui ont fait notre bonheur depuis le décollement du développement industriel ${ }^{12}$ "; la seconde quant à elle s'efforce de "revaloriser les liens qui nous attachent les uns aux autres ainsi qu'à notre environnement, ce qui implique de combattre nos addictions actuelles aux fétiches de la croissance consumériste ${ }^{13} »$.

Mais, qui est précisément dans chaque cas ce « nous » désignant ceux qui sont pris en compte comme dignes de relations respectueuses, de considération éthique voire de justice ? S'il ne tient compte dans le premier cas que d'une partie de l'humanité vivant aux dépens du reste ainsi que des milieux réduits au rang de ressources, la deuxième formule n'implique pas le même genre de relations intrinsèquement utilitaristes. Là où la gestion environnementale prétend trouver un point d'équilibre soutenable ou durable pour ne pas avoir à changer le paradigme de l'extraction des ressources, l'écologie radicale ou profonde pose comme prémisse un tout autre point d'équilibre : la possibilité de relations vivables et pacifiques pour l'ensemble des individus, espèces et milieux ; possibilité dont elle fait dépendre la pertinence toujours à réévaluer du développement technique et industriel.

La notion d'écologie apparaît tardivement à la fin du XIX ${ }^{\mathrm{e}}$ siècle. Elle est définie par Haeckel comme "science des relations de l'organisme avec l'environnement [qui] comprend au sens large, toutes les conditions d'existence ${ }^{14} »$. Toutefois, le souci et la connaissance des relations entre les êtres existent bien plus tôt. Et dès qu'existe la notion du foyer, de la maison, de l'oïkos grec, tout ne tourne-t-il pas déjà autour de la question d'énonciation suivante : comment se constituent ces "nous " relationnels, ensembles et sous-ensembles reliés par divers attachements, par lesquels seuls le vivant est à proprement parler "vivant "? Question que seules les humanités pouvaient poser, peut-être : qui parle, en écologie, de qui et à qui ?

11 Cf. Arne Naess, "The Shallow and the Deep, Long-Range Ecology Movement. A Summary ", Inquiry, 16 (1973), p. 95-100.

12 Yves Citton, Pour une écologie de l'attention, Paris, Seuil, 2014, p. 156.

13 Id., p. 160.

14 Ernst Haeckel, Generelle Morphologien der Organismen, Berlin, G. Reimer, 1866. 
L'on pense certes à la première personne du singulier qui porte si souvent l'expérience concrète et située d'un nombre restreint de lieux, le récit plus ou moins scientifique ou pittoresque d'un témoignage singulier qui demande à être mis en relation à d'autres surveys pour faire avancer la science naturaliste (première personne si décisive encore dans le Nature Writing où le topos de la solitude dans la nature sauvage s'ajoute à la teneur naturaliste). La connaissance de la terre et du vivant comporte ainsi une irréductible part locale et contingente, très variable en fonction des milieux et des écosystèmes spécifiques. Ainsi l'écologie s'est-elle historiquement déclinée par exemple en écologie littorale, en écologie andine ou en écologie de la Grande Barrière de corail.

Mais en tant qu'étude des relations, elle suppose une mise en commun, une mise en rapport, et donc, un changement d'échelles à même de pouvoir parler au nom d'un " nous " (qu'il s'agisse, selon les cas, de l'humanité, du vivant ou de la biosphère). L'enjeu est de pouvoir passer des cas particuliers (spécimens, individus), sinon aux lois, du moins aux connaissances empiriques générales. Cela demande d'élargir les échelles et points de vue pour s'élever en pensée, de l'écosystème le plus local et spécifique à la globalité d'un cosmos qui englobe cet écosystème parmi tous les autres ${ }^{15}$. Ces opérations épistémologiques fondamentales sur lesquelles reposent les sciences de l'environnement, sont à chaque fois autant d'opérations linguistiques et éthiques qui engagent fortement les humanités, pour autant que "science sans conscience est ruine de l'âme ${ }^{16}$ ». Du point de vue linguistique, épistémologique et éthique qu'entendent faire valoir les humanités, ce passage du " je " singulier au "nous " général, intrinsèque à toute pensée écologique, pose une infinité de questions.

Les «nous » dont sont tissés les multiples discours, écologiques ou supposés tels, à propos de l'humanité et de la Terre, prennent-ils tous également en compte hommes et femmes ? Blancs et personnes de couleur ? Pauvres et riches? Vieux et jeunes? Humains et non-humains? De quelles intersectionnalités entre "races ", classes, sexes, genres, âges et même espèces, sont-ils à chaque fois, explicitement ou non, les vecteurs ? Comment permettent-ils ou non de faire attention aux problèmes de justice environnementale, à l'existence des conflits autour des ressources et de leurs usages à toutes les échelles où ils apparaissent, aussi bien dans le jeu géopolitique de l'espace-monde globalisé que dans les concurrences territoriales et locales qui le concrétisent ? Comment les problèmes écologiques et leurs mises en discours explicitent-ils ou non les points de vue différents des " centres" qui consomment toujours plus et des "périphéries" qui les fournissent en matières premières tout en étant victimes d'externalités négatives?

15 Sur l'extension éthique, cf. Aldo Leopold, Almanach d'un comté des sables suivi de Quelques croquis, trad. Anna Gibson, Paris, GF, 2000.

16 François Rabelais, Pantagruel [1542], Paris, Gallimard, 1964, p. 137. 
Voici quelques-unes des questions que le croisement entre écologie et humanités a vocation à éclairer. À l'heure du grand récit de l'urgence climatique universelle, comment concilier la variété des points de vue singuliers et la nécessité d'une commune condition planétaire, en tenant compte de l'inégal accès aux ressources, de l'inégale répartition des risques, de l'inégale responsabilité historique et actuelle dans leurs causes, sinon en repensant les possibilités d'une pensée historicisante et contextualisante ${ }^{17}$ ? Comment l'insituable commun de l'humanité peut-il ne pas s'effacer derrière les calculs, les tris de la statistique et des conflits particularistes, qui semblent ruiner par la concrétude singulière de leurs enjeux immédiats, toute prétention à penser les rapports entre la Terre et l'humanité universelle ?

À mesure que peu à peu, "les radicaux et les gestionnaires font avancer ensemble les frontières de notre attention collective aux questions écologiques ${ }^{18}$ ", l'écologie, en ce qu'elle participe et procède de chaque culture, y bouleverse les langues. La circulation de ses mots, tels que s'en saisit la gouvernance, détourne son sémantisme par le biais d'un écoblanchiment (greenwashing), le réduisant à un label "vert " visant à atténuer en apparence les activités prédatrices qui s'en réclament. C'est pourquoi la notion d'écologie doit non seulement être réappropriée par des langues autres qu'officielles, publicitaires ou communicationnelles, mais plus particulièrement être repensée des humanités refondées en vue de cela même. L'écologie est fondamentalement affaire de mémoire, de langage et d'interprétation.

Loin de tout storytelling, la pensée écologique suppose l'implication concrète, éthique et existentielle des êtres et des choses dans les mots mêmes qui les désignent et permettent leurs relations. La grande question qu'elle pose aux humanités est celle de la traductibilité dans les termes d'un langage en partie à inventer, de tout ce qui, ne parlant certes pas, entre tout autant que les êtres parlants en relation avec ce qui l'environne ${ }^{19}$. L'enjeu est sans doute de chercher leur contact autrement qu'avec certaines méthodes scientifiques conquérantes qui s'étaient mises en devoir de faire parler la nature, laquelle lorsqu'elle est sommée de s'exprimer, "se tait sous la torture ${ }^{20}$ ". Une partie substantielle des questionnements écologiques concerne en d'autres termes la traduction, le langage et les humanités.

17 Cf. Ramachandra Guha et Joan Martínez-Alier, "L'environnementalisme des riches ", in Émilie Hache (éd.), Écologie politique. Cosmos, communautés, milieux, trad. Cyril Le Roy, Paris, Amsterdam, 2012, p. 51-66.

18 Yves Citton, op. cit., p. 160.

19 Comme l'a notamment posée Le contrat naturel de Michel Serres (Paris, Flammarion, 1990), la question revient notamment à se demander comment représenter ou donner la parole à tout ou partie du non-humain (la nature, les animaux, les écosystèmes, etc.).

20 Johann Wolfgang von Goethe, Maximen und Reflexionen, $\$ 498$, HA, t. 12, p. 434. Sur le prométhéisme et l'orphisme, cf. Pierre Hadot, Le voile d'Isis. Essai sur l'histoire de l'idée de Nature, rééd. Folio, Gallimard, 2008. 
$\mathrm{Au}$ fond, il s'agit ici de faire cheminer ensemble des disciplines et des facultés que l'histoire intellectuelle et académique, française en particulier, a persisté à séparer depuis le positivisme. Y a-t-il encore un sens à ce que les sciences de l'homme et celles de la nature se dévisagent à bonne distance, scindant notamment, et de façon particulièrement stérile, la pensée écologique en autant d'hémisphères disjoints, réputés ne jamais pouvoir sérieusement relever à la fois de l'écologie scientifique et de l'écologie politique ? Histoire, géographie, littérature, philosophie, arts et langues peuvent-ils être tenus à la marge des savoirs qu'impliquent l'une comme l'autre ? Certainement plus, dès lors qu'il s'agit de penser conjointement, "la nature » ou tout ce qui relève de cette appellation singulière, et non plus "l'Homme » mais la bigarrure infinie des diversités composant les sociétés humaines.

Qu'un dossier conçu en réseau trouve sa place dans la revue Essais n'a rien que de naturel, tant à cause du lien de filiation critique qui l'unit à la tradition humaniste, fortement interrogée dans les pages qui suivent, que par les textes qu'il réunit, voués par leur projet même à une forme ou une autre d'essayisme. C'est donc sans surprise que ce numéro fait par exemple écho à celui consacré en 2012 aux Marges de l'humain, prolongeant le questionnement de et sur l'humanisme ainsi que du supposé " propre de l'homme » et de ses frontières poreuses. Ce dossier résonne avec toute une constellation de projets et de travaux avec lesquels il a cheminé en bonne intelligence, tant du côté des sciences sociales que de l'écocritique ${ }^{21}$. De la pesée subtile du rôle des différentes cosmovisions dans la construction ou la destruction du monde en passe d'être entièrement découvert, à cette écriture caractéristique des essais autour des « herbes sauvages et inutiles ${ }^{22}$ ", le patronage de Montaigne a du reste tout à voir avec ce qui ne s'est certes pas nommé de toute date " écologie ", mais a bel et bien existé sous d'innombrables formes et d'autres noms partout où des femmes et des hommes se sont posé la question de leur interaction mutuelle (en synchronie) et de leur co-évolution (en diachronie) avec les milieux qu'ils habitent.

La nécessité existe de penser conjointement et de façon complexe, autrement dit sans écraser les singularités ${ }^{23}$, une humanité plurielle et une planète certes unique, commune, mais habitée par des mondes et des cosmovisions

21 Parmi les nombreux chantiers écocritiques partenaires, citons le projet "Écologie et littérature " des Universités d'Angers, de Nantes et du Mans, sur la transmission des savoirs scientifiques dans la fiction contemporaine hors des États-Unis (Écolitt, http://ecolitt.univ-angers.fr/ fr/index.html) ou le séminaire d'écopoétique de l'Université de Perpignan (www.ecopoeticsperpignan.com).

22 Michel de Montaigne, Essais, I, 8 [1572], in Les Essais, Paris, Gallimard, "Bibliothèque de la Pléiade ", 2007, p. 54.

23 Sur la nécessité de penser politiquement les liens entre économie, droit et éthique des biens spécifiques que sont l'eau, l'air et la terre, cf. Pierre Dardot et Christian Laval, Commun, essai sur la révolution au XXIe siècle, Paris, La Découverte, 2014. 
variés. L'enjeu est de ne pas exporter par ethnocentrisme telle ou telle distinction supposément radicale entre " homme » et " nature ", partage spécifiquement naturaliste, dans l'ensemble plus vaste des ontologies qui composent le " plurivers " et pour lesquelles quelque chose comme une " nature " n'est pas discernable des hommes ${ }^{24}$. Le chantier est immense et déjà entamé par tant d'autres, et nous y contribuons ici en réunissant huit textes qui se déploient dans des directions complémentaires, sans prétention à l'exhaustivité ni à un quelconque systématisme; des essais au sens plein du terme.

Des classiques chinois antiques aux poètes contemporains européens et américains, une première grande direction a consisté à relire des corpus littéraires et leurs interprétations dans le temps, à l'aune de la crise écologique et de la conscience qu'a pu en avoir une partie de l'humanité. L'autre versant du volume, plus ancré dans les sciences humaines et sociales, envisage à la fois l'histoire de l'écologie politique et l'évolution actuelle de la philosophie environnementale, l'écoféminisme, la justice environnementale et la proposition inédite d'un humanisme relationnel. Ces deux grands axes ne sauraient sans doute exister l'un sans l'autre.

C'est non sans lien avec le geste renaissant des humanistes que Gwennaël Gaffric et Jean-Yves Heurtebise relisent le Zhuangzi (庄子, IV et III siècles av. J.-C.), recueil chinois de textes philosophiques et classique du taoïsme, en interrogeant le regain d'intérêt qu'il suscite auprès des penseurs de l'éthique environnementale, tentés de le présenter comme antidote «nonoccidental " à une crise écologique essentiellement causée par l'Occident. Leur proposition consiste à le lire au contraire sans l'essentialiser comme issu d'une culture intrinsèquement différente voire exotique, afin de montrer ce qu'il apporte au-delà du canon national à l'éclairage d'une crise globalisée. Soustraire ce texte au schème d'une " pensée chinoise écologique " s'opposant trait pour trait à une " philosophie occidentale écocidaire " permet de ne pas se leurrer sur les effets de légitimation culturelle et littéraire qui prétendent instrumentaliser ce texte (comme d'autres) pour disposer de son aura.

Mathieu Perrot traduit et interprète des textes du poète américain Allen Ginsberg, figure centrale de la Beat Generation et pionnier du mouvement de la contre-culture américaine. Au fil de traductions inédites qui révèlent une voix prophétique, frappante de lucidité, il s'attèle à faire apparaître l'engagement politique et écopoétique visionnaire d'Allen Ginsberg en faveur de la promotion de la conscience écologique. Critique à l'égard de la civilisation industrielle et du capitalisme, l'écriture d'Allen Ginsberg en dénonce avec force les conséquences sociales et environnementales, et prône des manières d'habiter le monde plus respectueuses de l'humain et du monde naturel.

24 Sur les différentes ontologies (naturaliste, totémiste, animiste et analogiste) et leur rapport à l'écologie, cf. Philippe Descola, L'écologie des autres. Sur le perspectivisme et le multinaturalisme, cf. Eduardo Viveiros de Castro, Métaphysiques cannibales, trad. Oiara Bonilla, Paris, PUF, 2009. 
Fondé sur une lecture des poèmes de Kenneth White, l'essai de Frédéric Poupon montre la façon dont ces textes géopoétiques accueillent la présence du monde physique, donnant une voix à ce qui ne parle pas, décentrant le poète et à travers lui «l'Homme » si central de l'humanisme, pour en suggérer la fusion avec le " dehors». Il montre aussi comment ils ouvrent la voie à une réflexion éthique mais plus encore à une manière d'être au monde, suggérant que l'éthique environnementale ne prend corps qu'au sein de formes artistiques et langagières concrètement expérimentées et renouvelées.

Certains des essais réunis ici font dialoguer la littérature avec les représentations culturelles. Le concept métaphorique du "viol de la terre » est ainsi au cœur de l'article de Noémie Moutel, qui l'étaye comme motif décisif dans un corpus de textes anglais des siècles classiques. En s'appuyant notamment sur les travaux de Theodore Roszak, Jacques Derrida, Annette Kolodny et Carolyn Merchant, elle retrace les jalons d'une métaphore, la " terre-commefemme " et son viol, de longue date ancrée dans l'imaginaire de la conquête et de la possession consubstantielle des femmes et des terres, aux origines de l'économie libérale, coloniale et patriarcale.

Dans la même lignée, Jeanne Burgart Goutal interroge l'apport de l'écoféminisme à la réflexion et l'engagement écologiste. Elle montre qu'il s'agit d'un apport à la fois critique et constructif qui fait de l'écofeminisme non pas une simple branche de la pensée écologique, mais un outil, une clé d'analyse permettant de reformuler et de dépasser les solutions proposées et les façons dont les problèmes sont posés par les courants dominant la réflexion environnementale. Elle évoque notamment la particularité de l'analyse écofeministe de la réflexion portant sur les injustices environnementales, présente les griefs des tenants de l'écoféminisme comme Val Plumwood à l'égard des mouvements de l'écologie superficielle et de l'écologie profonde, et montre en quoi l'écoféminisme participe de la radicalisation des idéaux écologistes ${ }^{25}$.

En étudiant la prise en compte tardive des enjeux écologiques dans la politique espagnole à travers le frémissement des années 1980 dans le corps doctrinal et programmatique du Parti Socialiste Ouvrier Espagnol (PSOE), Vincent Marin illustre la façon dont les langages désynchronisés de la science, de la politique et de la culture compliquent l'entrée de visions du monde changeantes et complexes dans les pratiques sociales, fussent celles d'une "Transition démocratique " supposément dynamique, mais sous certains aspects sans doute assez figée.

25 Cf. aussi, pour une parution récente, Émilie Hache (choix et présentation), Reclaim. Recueil de textes écoféministes, Paris, Cambourakis, coll. "Sorcières ", 2016. 
En regard de ces faits et de leur analyse par les sciences sociales, les sciences humaines traitant d'écologie modèlent aussi des pensées plus spéculatives et des principes. C'est ainsi qu'à l'encontre d'un certain humanisme " toxique " où l'humain a été non seulement au centre du monde, mais sans relations avec lui, Baptiste Morizot dessine dans son essai philosophique les contours d'une éthique relationnelle où les relations existeraient avant les termes. C'est à partir des principaux textes engagés dans la discussion entre l'humanisme, la modernité et l'écologie, de Simondon à Bruno Latour, qu'il propose d'envisager l'homme à nouveaux frais comme un nœud de relations avec l'ensemble du vivant et de ses conditions non-vivantes.

Dans son essai, Blanchard Makanga propose quant à lui un recadrage normatif des sociétés industrielles et technoscientiques. Il évoque la nécessité d'encadrer éthiquement l'usage des technosciences pour éviter les actes relevant de ce qu'il nomme "l'incivisme environnemental ». Au lieu de se positionner pour ou contre les technosciences, il faut selon lui se positionner pour ou contre des usages. Il faut choisir des usages responsables, à la manière dont nous y invite Hans Jonas, pour qu'émerge une " civilisation environnementale " adaptée aux sociétés techniciennes contemporaines.

Complément à ces essais, notre entretien avec Catherine Larrère offre un tour d'horizon des problématiques qui marquent les débats en philosophie de l'écologie et en esquisse les enjeux majeurs pour le XXIe Siècle. Celle qui a contribué à promouvoir ces réflexions en France à partir des années 1990 apporte ici un éclairage sur les problématiques liées à l'éthique environnementale, aux inégalités environnementales et à l'écologie politique ${ }^{26}$.

Fabien Colombo Diplômé de philosophie, économie-sociologie et anthropologie Université Toulouse Jean Jaurès fabien.colombo@aol.fr

Nestor Engone Elloué Sciences, Philosophies, Humanités (EA 4574 SPH) Université Bordeaux Montaigne n.engonell@yahoo.fr

Bertrand Guest

Langues, Littératures, Linguistique, des Universités d'Angers et du Maine (EA 4335 3L.AM)

Université d'Angers

bertrand.guest@univ-angers.fr

26 Cf. aussi Catherine Larrère et Raphaël Larrère, Penser et agir avec la nature. Une enquête philosophique, Paris, La Découverte, 2015. 